\title{
Modelling hadronic interactions in cosmic ray Monte Carlo generators
}

\author{
Tanguy Pierog ${ }^{\mathrm{a}}$ \\ Karlsruhe Institute of Technology (KIT), IKP, 76021 Karlsruhe, Germany
}

\begin{abstract}
Currently the uncertainty in the prediction of shower observables for different primary particles and energies is dominated by differences between hadronic interaction models. The LHC data on minimum bias measurements can be used to test Monte Carlo generators and these new constraints will help to reduce the uncertainties in air shower predictions. In this article, after a short introduction on air showers and Monte Carlo generators, we will show the results of the comparison between the updated version of high energy hadronic interaction models EPOS LHC and QGSJETII-04 with LHC data. Results for air shower simulations and their consequences on comparisons with air shower data will be discussed.
\end{abstract}

\section{Introduction}

Knowing the elemental composition of cosmic ray particles arriving at Earth is of crucial importance to understand the production and propagation of cosmic rays. Unfortunately, cosmic rays can be measured only indirectly above an energy of $10^{14} \mathrm{eV}$ through the cascades of secondary particles, called extensive air-showers (EAS), that they produce in the atmosphere (for a recent review, see [1]). Only by simulating the generation of EAS and comparing the predictions with measurements can one draw conclusions on the primary mass composition of the arriving particles [2]. With the operation of modern large-scale experiments, the reliability of airshower simulations has become the source of the largest systematic uncertainty in the interpretation of cosmic-ray data [3-8]. While the electroweak interaction processes are reasonably well understood, modelling of hadronic multi-particle production is subject to large theoretical uncertainties that are, moreover, difficult to estimate [9-11].

The Large Hadron Collider (LHC) at the CERN laboratory allows us to access, for the first time, the energy region above the cosmic ray spectral knee in the laboratory (Fig. 1). Therefore an analysis of inclusive particle data taken at the LHC is particularly interesting for constraining existing hadronic interaction models and for testing possible new mechanisms of hadron production [13]. Data from LHC experiments published so far have mostly been taken with detectors covering the central phase space region in pseudorapidity $(|\eta|<2.5)$. This region is most easily accessible in collider experiments and is also the region of the highest rapiditydensity of produced particles. The first data have been compared to cosmic ray models in [14]. On the other hand, since the number of particles in an air-shower is roughly proportional to the energy of the primary particle, the most energetic outgoing particles of an interaction, emitted in the very forward region of a collider experiment - such as in diffractive interactions - are the most important ones for understanding air-showers.

In this paper, we will discuss changes in the hadronic model predictions after LHC data and their consequences on air shower observables. In the first section, we will explain the so-called Heitler model to extract from a simple toy model the main hadronic observables which drive the development of air showers. We will then compare the results of the hadronic interaction models with LHC data for such observables. Using detailed Monte Carlo simulations done with CONEX [15], the new predictions for $\mathrm{X}_{\max }$, for the number of muons, and for the missing energy will be presented. Finally we will take the example of the muon production depth (MPD) measured by the Pierre Auger Observatory [7] to see how air shower measurements can constrain hadronic interaction physics.

\section{Heitler's model}

To qualitatively describe the dependence of shower development on some basic parameters of particle interaction, decay and production, a very simple toy model can be used. Although initially developed for electromagnetic (EM) showers [16] it can also be applied to hadronic showers [17].

\subsection{Electromagnetic showers}

For simplicity, instead of having three particle types $(\gamma$, $e^{+}$and $\left.e^{-}\right)$like in electromagnetic showers, we will consider only one particle with energy $E$ with only one EM interaction producing two new particles with energy $E / 2$ after a fixed interaction length of $\lambda_{e}$, see Fig. 2 lefthand side.

Denoting with $n$ the number of generations (consecutive interactions), the number of particles at a given depth $X=n \cdot \lambda_{e}$ follows from

$$
N(X)=2^{n}=2^{X / \lambda_{e}},
$$

This is an Open Access article distributed under the terms of the Creative Commons Attribution License 4.0, which permits unrestricted use, distribution, and reproduction in any medium, provided the original work is properly cited. 


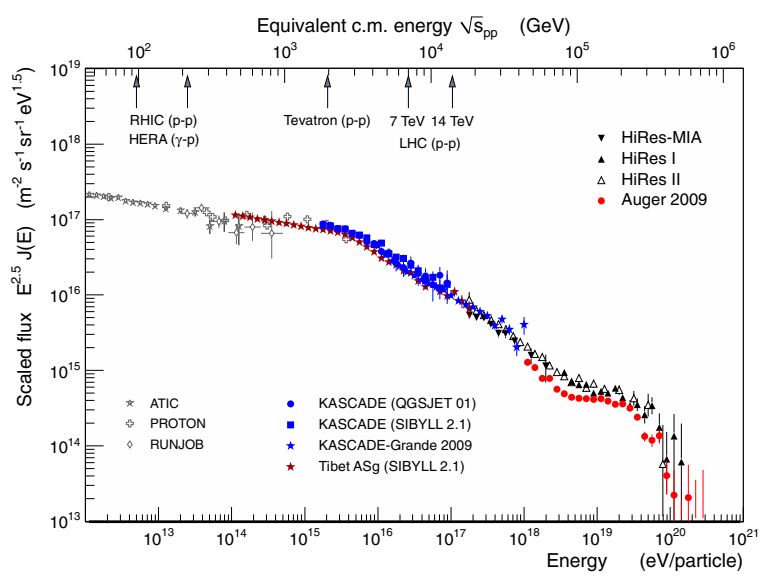

Figure 1. Flux of cosmic rays arriving at Earth rescaled by the energy to the power 2.5. Data references are given in [12].
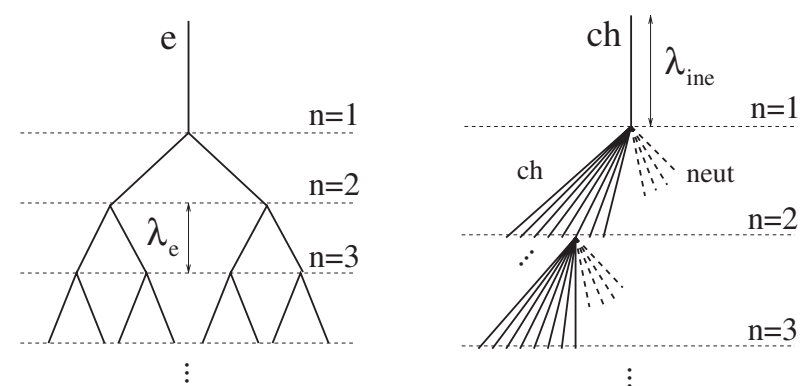

Figure 2. Schematic view of electromagnetic cascades (lefthand side) and schematic view of hadronic cascades (right-hand side). Dashed lines represent neutral particles $\left(\pi^{0}\right)$ and solid lines charged particles $\left(\pi^{ \pm}\right)$. Only one charged hadron interaction is shown for each generation.

with the energy $E$ per particle for a given primary energy $E_{0}$ being

$$
E(X)=\frac{E_{0}}{2^{X / \lambda_{e}}} .
$$

Defining the critical energy $E_{c}(\sim 85 \mathrm{MeV}$ in air $)$ as the energy below which energy loss processes dominate over particle production, one can make the assumption that the shower maximum is reached at a depth at which the energy of the secondary particles reaches $E_{c}$. Then two main shower observables are given by

$$
N_{\max }=\frac{E_{0}}{E_{c}} \quad \text { and } \quad X_{\max }^{e}\left(E_{0}\right) \sim \lambda_{e} \cdot \ln \left(\frac{E_{0}}{E_{c}}\right) .
$$

This simplified picture does not reproduce the detailed behavior of an EM shower, but two important features are well described: the number of particles at shower maximum is proportional to $E_{0}$ and the depth of shower maximum depends logarithmically on the primary energy $E_{0}$.

\subsection{Hadronic showers}

Generalizing this idea, a hadronic interaction of a particle with energy $E$ is assumed to produce $n_{\text {tot }}$ new particles with energy $E / n_{\text {tot }}$, two thirds of which are charged particles $n_{\mathrm{ch}}$ (charged pions) and one third are neutral particles $n_{\text {neut }}$ (neutral pions), as shown Fig. 2 righthand side. Neutral particles decay immediately into EM particles $\left(\pi^{0} \rightarrow 2 \gamma\right)$. After having traveled a distance corresponding to the mean interaction length $\lambda_{\text {ine }}$, charged particles re-interact with air nuclei if their energy is greater than some typical decay energy $E_{\mathrm{dec}}$.

\subsubsection{Energy transfer}

In each hadronic interaction, one third of the energy is transferred to the EM shower component. After $n$ generations the energies in the hadronic and EM components are given by

$$
\begin{gathered}
E_{\mathrm{had}}=\left(\frac{2}{3}\right)^{n} E_{0} \\
E_{\mathrm{EM}}=\left[1-\left(\frac{2}{3}\right)^{n}\right] E_{0},
\end{gathered}
$$

where $n$ will be calculated later. Simulations show that the total number of generations before a vertical shower reaches the ground level is typically about 5 to 6 [18].

Even in an air shower initiated by a hadron, most of the energy is carried by EM particles ( $\sim 90 \%$ for $n=6)$. Hence the depth of shower maximum is given by that of the EM shower component, $X_{\max }^{e}$. As the first hadronic interaction produces EM particles of energy $\sim E_{0} / n_{\text {tot }}$ one gets

$$
\begin{gathered}
X_{\max }\left(E_{0}\right) \sim \lambda_{\text {ine }}+X_{\text {max }}^{e}\left(E_{0} / n_{\text {tot }}\right) \\
\sim \lambda_{\text {ine }}+\lambda_{e} \cdot \ln \left(\frac{E_{0}}{n_{\text {tot }} E_{c}}\right),
\end{gathered}
$$

where $\lambda_{\text {ine }}$ is the hadronic interaction length. This simplified expression for the depth of maximum neglects the EM sub-showers initiated by hadrons of later generations. The inclusion of higher hadronic generations does not change the structure of Eq. (7), only the coefficients change (see, for example, [19]).

\subsubsection{Muon component}

To keep the picture simple, we assume that all charged hadrons decay into muons when their energy reaches $E_{\text {dec }}$. In a real shower, this limit can be seen as the characteristic energy where interaction length and decay length of charged pions are similar (about $150 \mathrm{GeV}$ for pions). By construction, charged particles will reach the energy $E_{\mathrm{dec}}$ after $n$ interactions

$$
E_{\mathrm{dec}}=\frac{E_{0}}{\left(n_{\mathrm{tot}}\right)^{n}} .
$$

Since one muon is produced in the decay of each charged particle, we get

$$
N_{\mu}=n_{\mathrm{ch}}^{n}=\left(\frac{E_{0}}{E_{\mathrm{dec}}}\right)^{\alpha},
$$

with $\alpha=\ln n_{\mathrm{ch}} / \ln n_{\text {tot }}=1+\ln R / \ln n_{\text {tot }} \approx 0.82 \ldots 0.95$ [19] where $R=n_{\mathrm{ch}} / n_{\text {tot }}<1$. The number of muons 
produced in an air shower depends not only on the primary energy and air density, but also on the total particle multiplicities and in a much more sensitive way [20] of the charged over all-particle ratio of hadronic interactions.

It should be kept in mind that the parameters of the model are only effective quantities and are not identical to the respective quantities measured at accelerators. In particular, the approximation of all secondary particles carrying the same energy is only motivated by the fact that it allows us to obtain simple, closed expressions. The wellknown leading particle effect, typically quantified by the (in)elasticity of an interaction, can be implemented in the model [17] but will not be considered here.

\section{Hadronic Interaction models and LHC data}

It is clear that such a toy-model, as described in the previous section, only gives a very much over-simplified account of air shower physics. However, the model allows us to qualitatively understand the dependence of many air shower observables on the characteristics of hadronic particle production. Accordingly the parameters of hadron production which are most important for air shower development are the cross section (or mean free path), the multiplicity of secondary particles of high energy, and the production ratio of neutral to charged particles. Until the start of the LHC, these parameters were not well constrained by particle production measurements at accelerators. As a consequence, depending on the assumptions of how to extrapolate existing accelerator data, the predictions of hadronic interaction models differ considerably.

There are several hadronic interaction models commonly used to simulate air showers. Here we will focus on the two high energy models which were updated to take LHC data at $7 \mathrm{TeV}$ into account: QGSJETII-03 [21,22] changed into QGSJETII-04 [23] and EPOS 1.99 [24, 25] was replaced by EPOS LHC (V3400), both available since CORSIKA V7.3700 [26]. There is no major change in these models but in addition to some technical improvements, some parameters were changed to reproduce TOTEM [27] cross sections. Both are based on Gribov-Regge multiple scattering, perturbative QCD and string fragmentation. The former versions reproduce accelerator data and even first LHC data reasonably well ([14] and Figs. 8 and 9) but predict different extrapolations above $E_{\mathrm{cms}} \sim 1.8 \mathrm{TeV}$ center-of-mass (cms) energy ( $E_{\text {lab }} \sim 10^{15} \mathrm{eV}$ kinetic energy) that lead to very different results at high energy $[2,28]$ which can be improved using LHC data.

\subsection{EPOS model}

EPOS LHC [29] is a minimum bias monte-carlo hadronic generator used for both heavy ion interactions and cosmic ray air shower simulations. It is based on EPOS 1.99 [24,25] retuned to reproduce LHC data on a higher precision level. As for most of high energy hadronhadron interaction models, it is based on the simple parton model which can be seen as an exchange of a "parton ladder" between the two hadrons.

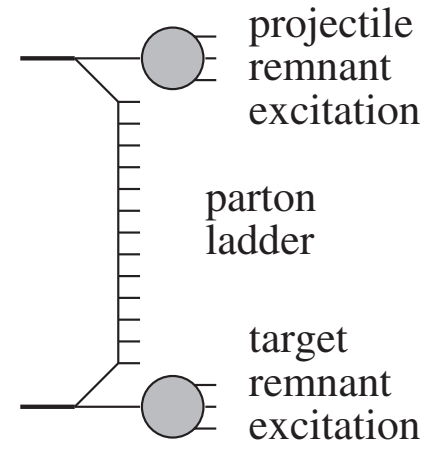

Figure 3. Elementary parton-parton scattering: the hard scattering in the middle is preceded by parton emissions attached to remnants. The remnants are an important source of particle production even at intermediate energies $(\sim 100 \mathrm{GeV} \mathrm{cms})$.

In EPOS, the term "parton ladder" is actually meant to contain two parts [30]: the hard one, as discussed above, and a soft one, which is a purely phenomenological object, parameterized in Regge pole fashion. This is the so called Pomeron used as elementary parton-parton interaction in EPOS.

In additions to the parton ladder, there is another source of particle production: the two off-shell remnants, see Fig. 3. We showed in Ref. [31] that this "three object picture" can solve the "multi-strange baryon problem" of conventional high energy models, see Ref. [32].

Hence EPOS is a consistent quantum mechanical multiple scattering approach based on partons and strings [30], where cross sections and the particle production are calculated consistently, taking into account energy conservation in both cases (unlike other models where energy conservation is not considered for cross section calculations [33]). The main consequence of this energy sharing process is that the number of Pomerons generated event-by-event does not follow a simple Poissonian distribution. As a consequence it is much less probable to produce events with a very large number of Pomerons (large multiplicity) compared to the standard Gribov-Regge approach like in QGSJETII. Nuclear effects related to Cronin transverse momentum broadening, parton saturation, and screening have been introduced into EPOS [24]. Furthermore, high density effects leading to collective behavior in heavy ion collisions are also taken into account [34].

Thanks to a Monte Carlo, first the collision configuration is determined: i.e. the number of each type of Pomerons exchanged between the projectile and target is fixed and the initial energy is shared between the Pomerons and the two remnants. Then particle production is accounted from two kinds of sources, remnant decay and cut Pomeron. A Pomeron may be regarded as a twolayer (soft) parton ladder attached to projectile and target remnants through its two legs. Each leg is a color singlet, of type q $\bar{q}$, qqq or $\overline{q q q}$ from the sea, and then each cut Pomeron is regarded as two strings, cf. Fig. 4a) and b).

It is a natural idea to take quarks and anti-quarks from the sea as string ends for soft Pomeron in EPOS, because an arbitrary number of Pomerons may be involved.

Thus, besides the three valence quarks, each remnant has additionally quarks and anti-quarks to compensate the 
a)

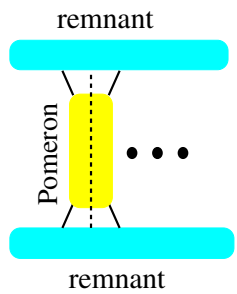

c)

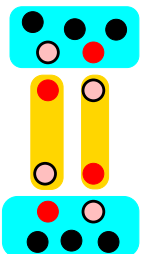

b)
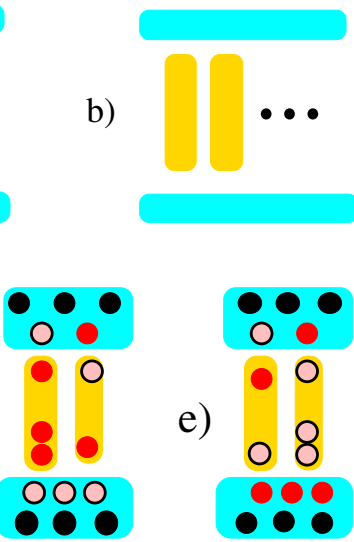

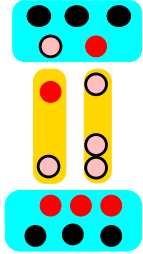

Figure 4. a) Each cut Pomeron is regarded as two strings b). c) The most simple and frequent collision configuration has two remnants and only one cut Pomeron represented by two $q-\bar{q}$ strings. d) One of the $\bar{q}$ string ends can be replaced by a qq string end. e) With the same probability, one of the q string ends can be replaced by a $\overline{\mathrm{qq}}$ string end.

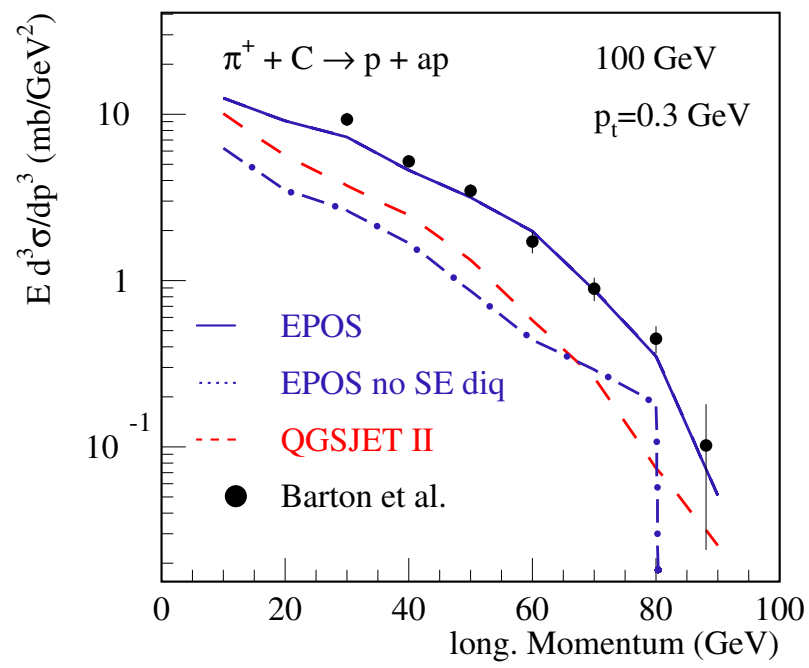

Figure 5. Model comparison: longitudinal momentum distributions of pion carbon collisions at $100 \mathrm{GeV}$ from EPOS with (full) or without (dashed-dotted) sting-end diquarks and QGSJETII (dashed) compared to data [35].

flavors of the string ends, as shown in Fig. 4c). According to its number of quarks and anti-quarks, to the phase space, and to an excitation probability, a remnant decays into mesons and/or (anti)baryons [31]. Furthermore, this process leads to a baryon stopping phenomenon in which the baryon number can be transferred from the remnant to the string ends (for instance in $4 \mathrm{~d}$ ), depending on the process, the $3 \bar{q}+3 q$ can be seen as 3 mesons or a baryonantibaryon pair).

In the case of a meson projectile, this kind of diquark pair production at the string ends leads to an increase of the (anti)baryon production in the forward production in agreement with low energy pion-nucleus data [35] as shown Fig. 5. Comparing to QGSJETII model which do not have diquark as string ends or using only $\mathrm{q} \overline{\mathrm{q}}$ as string end in EPOS, we can clearly see that this process is needed

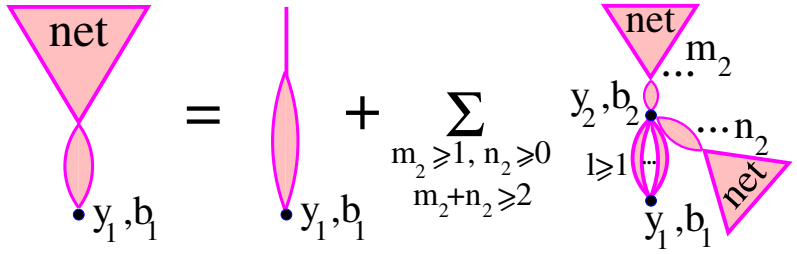

Figure 6. Enhanced fan diagrams as used in QGSJETII [37].

to reproduce experimental data. As a consequence it is part of the larger number of muons in air shower simulations with EPOS.

Energy momentum sharing and remnant treatment are the key points of the model concerning air shower simulations because they directly influence the multiplicity and the inelasticity of the model.

\subsection{QGSJETII model}

QGSJETII-04 [23] model is a minimum bias hadronic interaction model optimized for air shower simulations. It has a minimum set of parameters to reduce the uncertainty due to the extrapolation to high energy and as a consequence has a less detailed description of the final stage of hadronic interactions (no final state effect, no rare particle production, etc. which limit the data set to which it can be compared). It is the current last evolution of the series of models based on Quark-Gluon and Strings model with Jet [36].

The elementary interaction used in QGSJETII is a semi-hard Pomeron very similar to the one used in EPOS. But to take into account non-linear effects at high energy, a very complex resummation scheme has been developed to take into account any type of Pomeron-Pomeron interactions resulting in a net fan diagram (see Fig. 6) which can be used for both cross-section and particle production. But then only the standard Gribov-Regge type of calculation can be used, meaning that energy sharing is not taken into account in cross-section calculation and when the total number of multiple scattering is calculated.

Like in EPOS, QGSJETII has some remnant from the projectile and target but in a simplified scheme which does not allow more than one quark exchange with the string ends. In particular diquarks are not allowed as string ends which gives quite different forward baryon production compared to EPOS as shown Fig. 5. So even if the two models are based on a very similar approach (parton ladder and Gribov-Regge based multiple interactions), the detailed treatments of energy sharing, non-linear effects, final state nuclear effects and remnant production lead to different extrapolations in both proton and pion interactions and thus for air shower observables as shown in the next sections.

\subsection{Cross section}

As shown in Eq. (7), the cross section is very important for the development of air showers and in particular for the depth of shower maximum. As a consequence, the number of electromagnetic particles at ground level is strongly correlated to this observable (if the shower maximum is closer to ground, the number of particles is higher). 


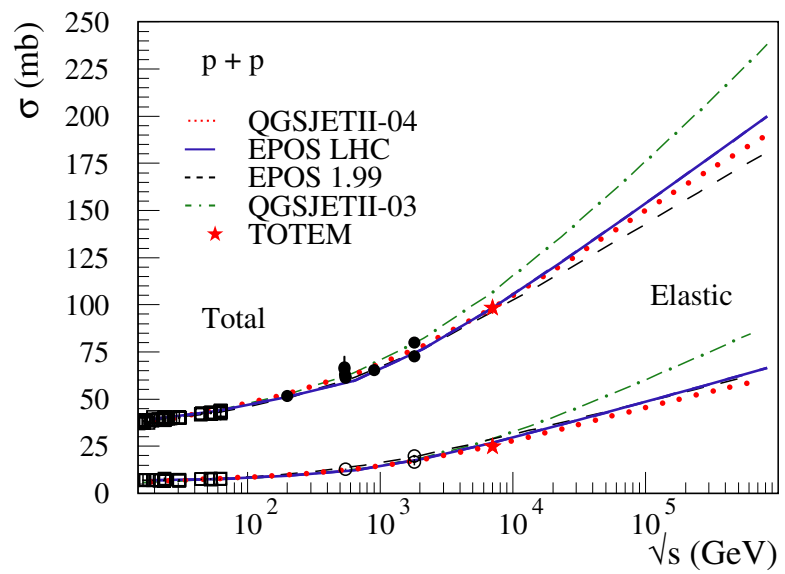

Figure 7. Total and elastic p-p cross sections calculated with EPOS LHC (full line), QGSJETII-04 (dotted line), EPOS 1.99 (dashed line) and QGSJETII-03 (dashed-dotted line). Points are data from [39] and the stars are the LHC measurements by the TOTEM experiment [27].

The total cross section of proton-proton scattering is usually used as an input to fix basic parameters in all hadronic interaction models. Therefore it is very well described by all the models at low energy, where data exist [38]. And then it diverges above $2 \mathrm{TeV}$ cms energy because of different model assumptions. As shown on Fig. 7 the new point measured by the TOTEM experiment at $7 \mathrm{TeV}$ reduces the difference between the models by a factor of 5 (50 to $10 \mathrm{mb})$. In all the figures EPOS LHC is represented by a full (blue) line, QGSJETII-04 by a dotted (red) line, EPOS 1.99 by a dashed (black) line and QGSJETII-03 by a dashed-dotted (green) line.

\subsection{Multiplicity}

According to Eq. (7), the multiplicity plays a similar kind of role as the cross section, but with a weaker dependence $(\log )$. On the other hand, the predictions from the models had much larger differences for the multiplicity compared to the cross section. As shown in Fig. 8 left-hand side, the particle density at mid-rapidity is well reproduced by all the models up to $2 \mathrm{TeV}$ where Tevatron data [40] constrain the results, but at the highest energies in $\pi$-air, the difference can be as high as a factor of 10 (Fig. 8 right-hand side). After re-tuning at $7 \mathrm{TeV}$ to be compatible with CMS data [41] or ALICE data [42], in Fig. 9, the difference, even at high energy in $\pi$-air, is less than a factor of 2. On the right-hand side of Fig. 9, we can see that not only the averaged multiplicity had been changed after re-tuning, but the fluctuations are now very similar for QGSJETII-04 and EPOS LHC. This will be important for the fluctuations of the air shower maximum.

So, for both cross section and multiplicity, when the models are constrained by LHC data up to $7 \mathrm{TeV}$, the extrapolation to the highest energy is not so different any more. This will have a strong impact on $\mathrm{X}_{\max }$ uncertainty in air shower simulations.

\subsection{Baryon production}

Another important observable for EAS is the number of muons reaching the ground. Using Eq. (9) and the definition of $\alpha$ and $R$, it has been shown in [20] that the number of (anti)baryons plays an important role in the value of $R$ especially if we take into account the leading particle effect. As a consequence the number of muons in EAS is sensitive to the number of (anti)baryons produced in the hadronic interactions and it is important to check the production of such particles in LHC data.

Both ALICE [43] and CMS [44] experiments published very nice results on identified spectra used to constrain models used for air shower simulations. As shown in Fig. 10, these data help a lot to reduce the differences between the models especially because they resolve an ambiguity on the phase space used to produce the anti-proton over pion ratio as a function of the number of charged particles with Tevatron data at $1.8 \mathrm{TeV}$. LHC data are much better defined and can be used to constrain the production of baryon pairs at mid-rapidity (largely dominated by string fragmentation).

It is important to notice that not only (and not all) (anti)baryons enter into the definition of the ratio $R$. All particles which do not decay into an electromagnetic particle can play a similar role and keep the energy of the shower into the hadronic channel to produce muons. For instance in QGSJETII-04 the newly introduced $\rho^{0}$ resonance as excited state of the pion remnant in pion interactions has a very strong influence on the muon production. Since forward $\pi^{0} \mathrm{~s}$, transferring a lot of energy in the electromagnetic channel, are replaced by particles which decay into charged pions, the energy is kept in the hadronic channel. This is clearly illustrated by Fig. 11 where we can see that QGSJETII-04 reproduce nicely both $\pi^{0}$ and $\rho^{0}$ forward spectra while QGSJETII-03 producing no $\rho^{0}$ had to hard $\pi^{0}$ spectra. This effect was already in EPOS 1.99, being one source of difference between the 2 models. EPOS 1.99 model seems to underestimate $\rho^{0}$ production but this is due to a wrong mixing with $\omega$ resonance and, in fact, the sum of the 2 is correct. It has been corrected in EPOS LHC. On the other hand, in EPOS 1.99 another process producing forward (anti)baryons was missing at high energy and has been added in EPOS LHC. As a consequence the reduced rate of (anti)baryon production at mid-rapidity is compensated by more forward (anti)baryon production which is even more important for muon production. Unfortunately there is very little data to constrain this production channel especially in collider experiments. NA61 [47] and LHCf [48] experiments may help to constrain this process in the future.

\section{EAS simulations}

Using the air shower simulation package CONEX and the new versions of the high-energy hadronic interaction models, we can get an estimate of the resulting uncertainties.

In the following EAS simulation, results using EPOS LHC (V3400) and QGSJETII-04 are presented and compared to former results using QGSJETII-03 and EPOS 1.99.

As shown in Fig. 12, the mean depth of shower maximum, $X_{\max }$, for proton and iron induced showers simulated with CONEX is still different for EPOS LHC and QGSJETII-04. But now the elongation rate (the slope of the mean $X_{\max }$ as function of the primary 

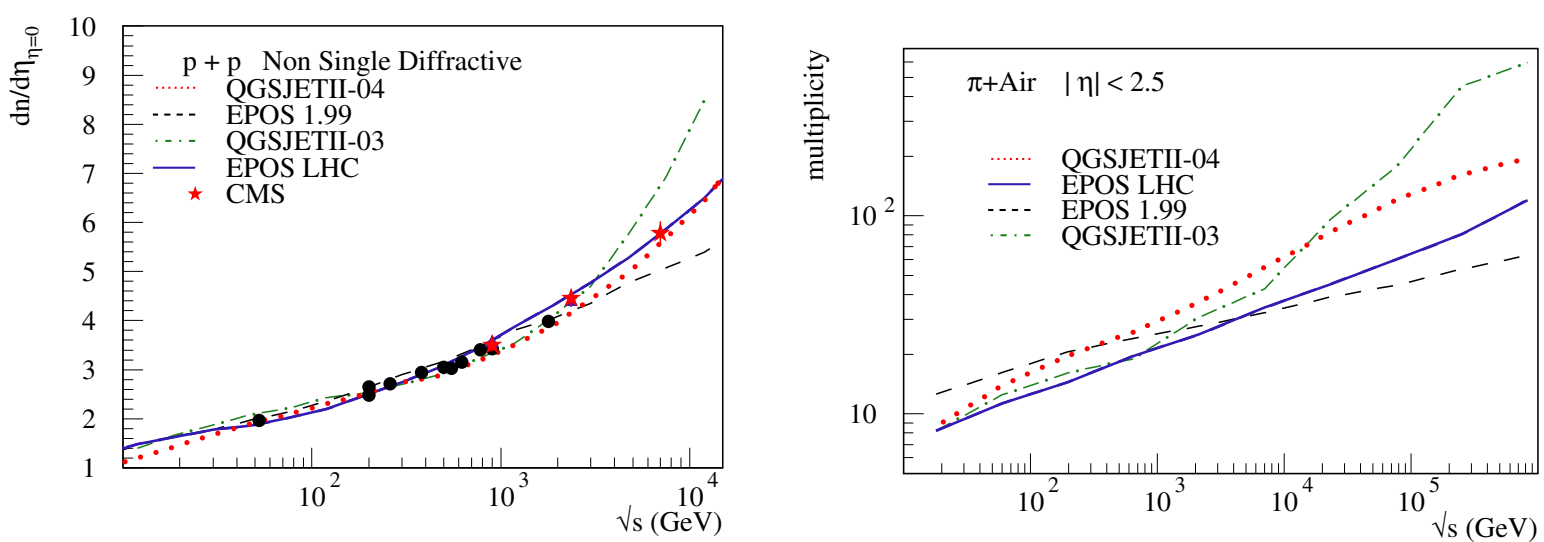

Figure 8. Particle density at $\eta=0$ for non single diffractive events (NSD) (left-hand side) and multiplicity for $|\eta|<2.5$ of $\pi$-air collisions (right-hand side) as a function of center of mass energy. Simulations are done with EPOS LHC (full line), QGSJETII-04 (dotted line), EPOS 1.99 (dashed line) and QGSJETII-03 (dashed-dotted line). Points are data from old experiments and red stars are from CMS experiment [41].
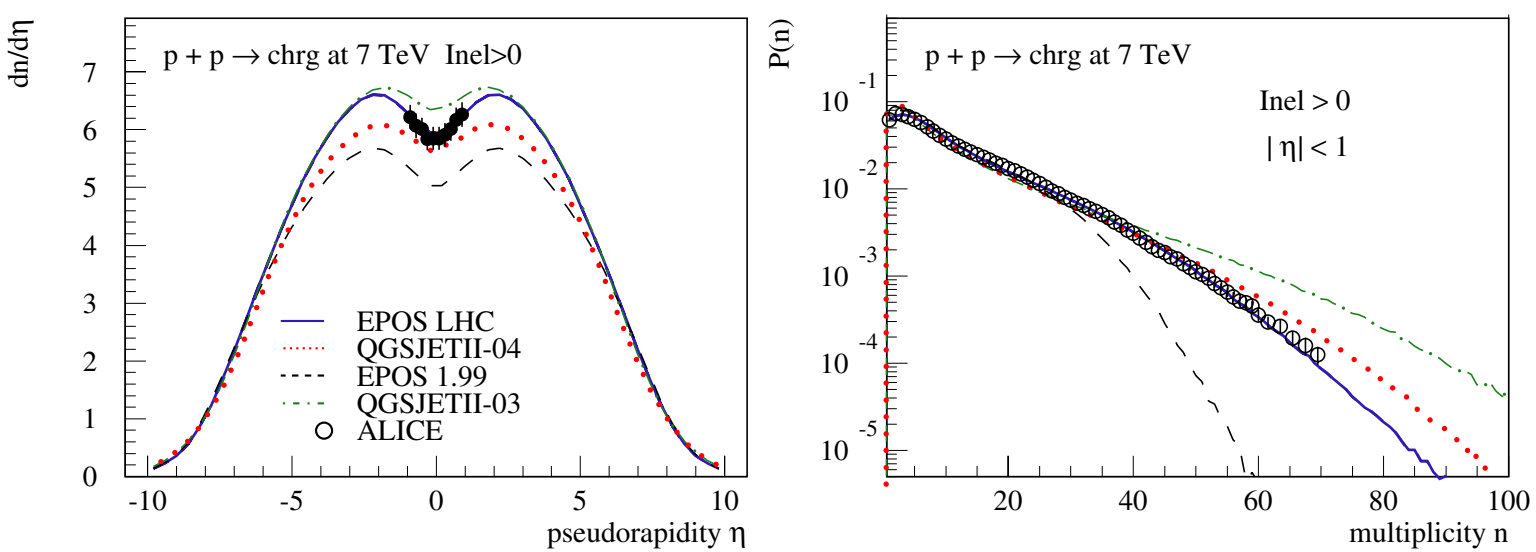

Figure 9. Pseudorapidity distribution $d N / d \eta$ for events with at least one charged particle with $|\eta|<1$ (left-hand side) and corresponding multiplicity distribution (right-hand side) for p-p interactions at $7 \mathrm{TeV}$. Simulations with EPOS LHC (full line), QGSJETII-04 (dotted line), EPOS 1.99 (dashed line) and QGSJETII-03 (dashed-dotted line) are compared to data points from the ALICE experiment [42].
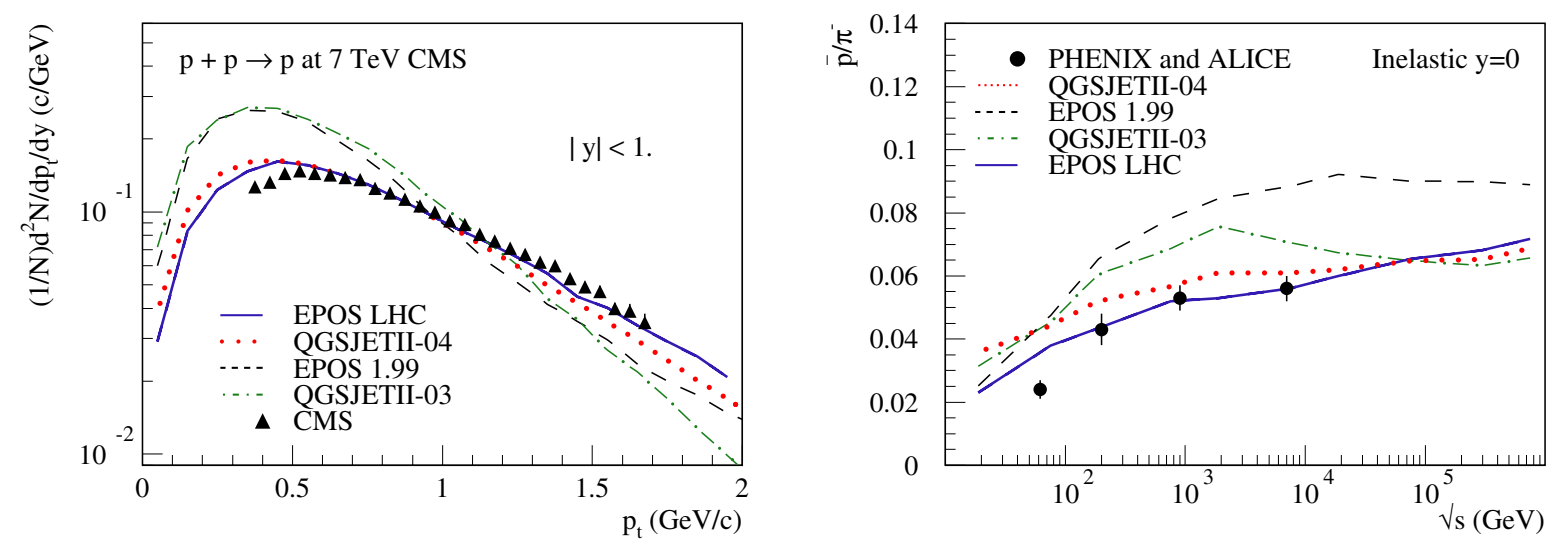

Figure 10. Transverse momentum distribution for $|y|<1$ for non single diffractive (NSD) p-p scattering at 7 TeV (left-hand side) and anti-proton over pion ratio at $y=0$ for p-p collisions (right-hand side) as a function of center of mass energy. Simulations are done with EPOS LHC (full line), QGSJETII-04 (dotted line), EPOS 1.99 (dashed line) and QGSJETII-03 (dashed-dotted line). Points are data from ALICE and PHENIX [43] experiments and triangles are from CMS experiment [44]. 

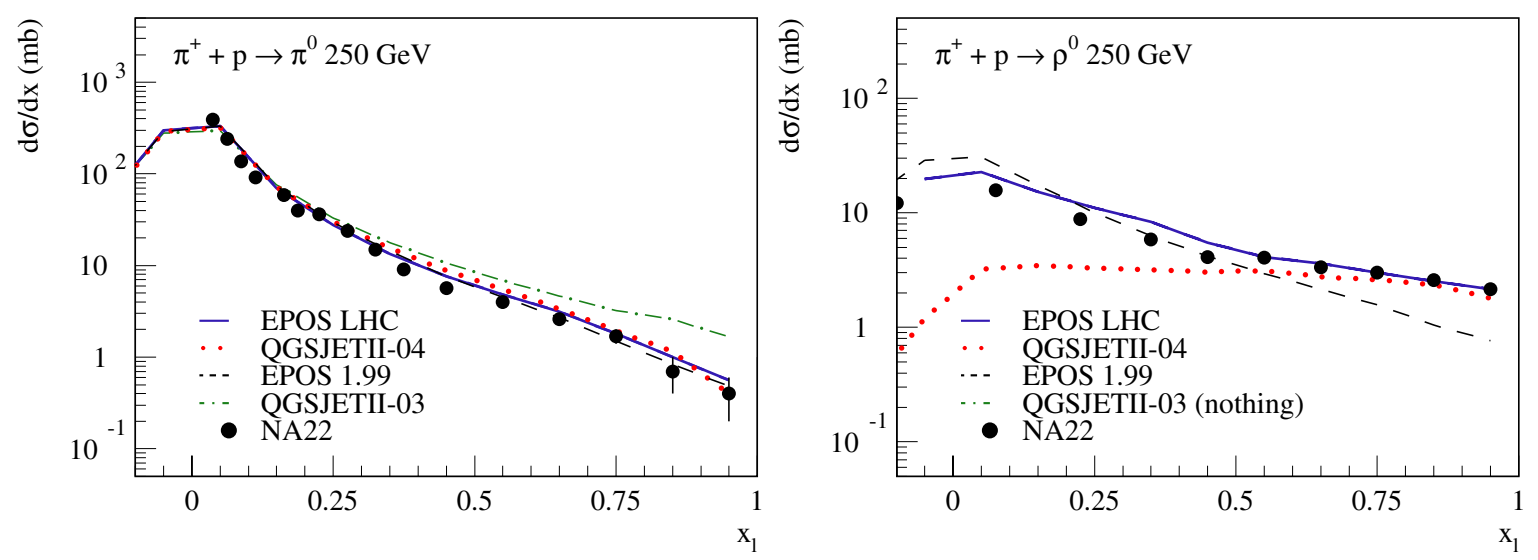

Figure 11. $\pi^{0}$ longitudinal momentum fraction distribution (left-hand side) and $\rho^{0}$ longitudinal momentum fraction distribution (righthand side) for $\pi$-p interactions at $250 \mathrm{GeV}$. Simulations with EPOS LHC (full line), QGSJETII-04 (dotted line), EPOS 1.99 (dashed line) and QGSJETII-03 (dashed-dotted line) are compared to data points from NA22 experiment $[45,46]$.

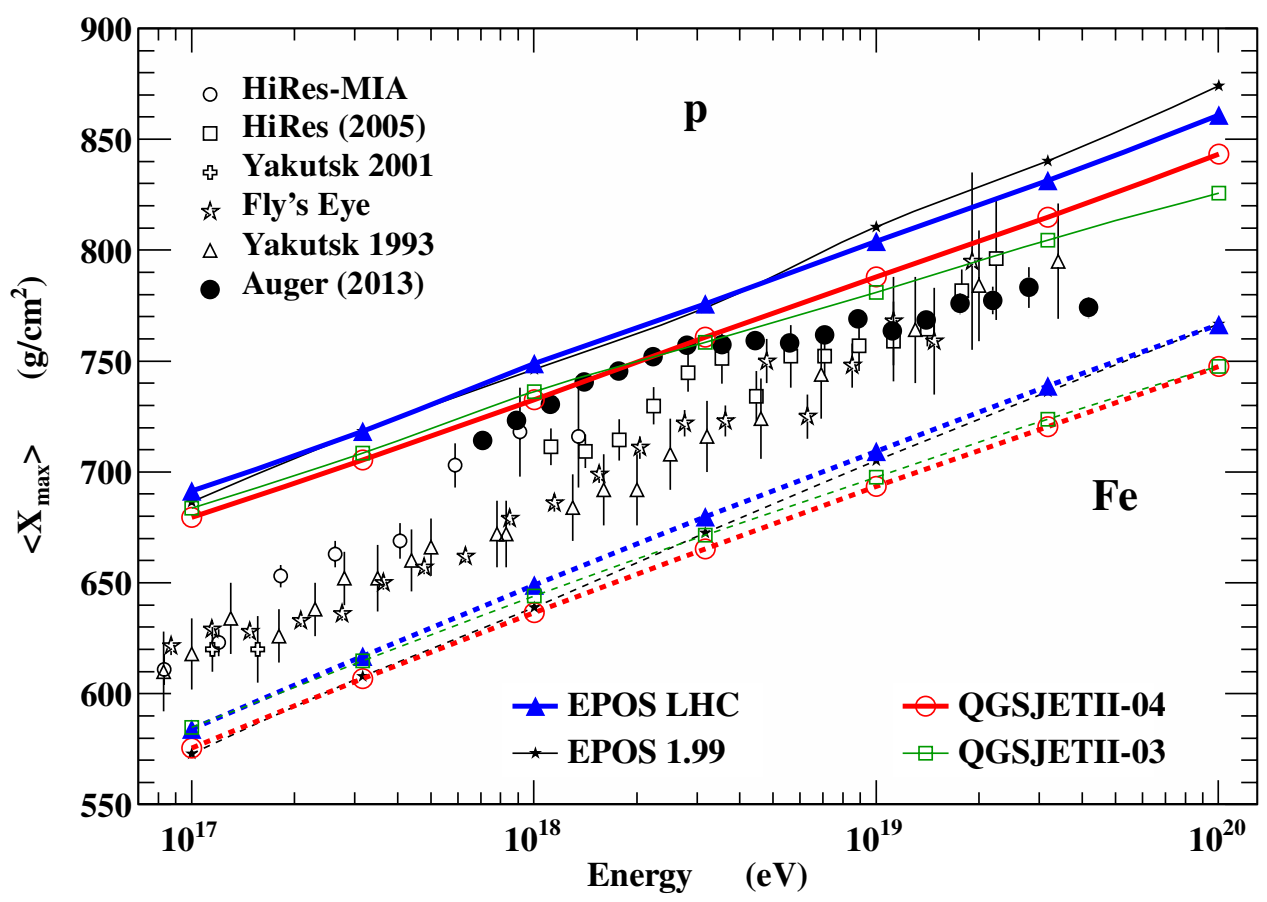

Figure 12. Mean $X_{\max }$ for proton and iron induced showers as a function of the primary energy. Predictions of different high-energy hadronic interaction models, full lines for proton and dashed lines for iron with full triangles for EPOS 1.99, open squares for QGSJETII-04, open circles for QGSJETII-03, and full stars for EPOS LHC, are compared to data. Refs. to the data can be found in [1] and [7].

energy) is the same in both cases while EPOS 1.99 had an elongation rate larger than QGSJETII-03. The difference between the two models is a constant shift of about $20 \mathrm{~g} / \mathrm{cm}^{2}$ (close to the experimental systematic error in Pierre Auger Observatory [7]) while before the difference was increasing up to $50 \mathrm{~g} / \mathrm{cm}^{2}$ at the highest energies.

This is very important to the study of the primary cosmic ray composition. If the models converge to a similar elongation rate, it will allow us to have a more precise idea on possible changes in composition at the "ankle" for instance where the Pierre Auger Observatory measures a break in the elongation rate of the data.

Concerning the number of muons at ground (for $40^{\circ}$ inclined showers at the height of $1500 \mathrm{~m}$ ), the difference between the new QGSJETII-04 and the old QGSJETII03 is even more impressive. We can see on Fig. 13 that QGSJETII-04 predicts now only 7\% less muons than EPOS 1.99, which is about $20 \%$ more than QGSJETII03. It is due to the change in baryon, strangeness and mostly resonance production as described in section 3.5. Concerning the predictions of EPOS LHC, the number of muons is very similar to the one in EPOS 1.99 because of the leading baryon production compensating the reduction of (anti)baryon production at mid-rapidity. So, even if the number of muons is much more similar now for the two most recent hadronic models, there is still an uncertainty of about $10 \%$ and furthermore the energy spectrum of the muons at ground is different between the models. This can be an important factor for the attenuation length of the 


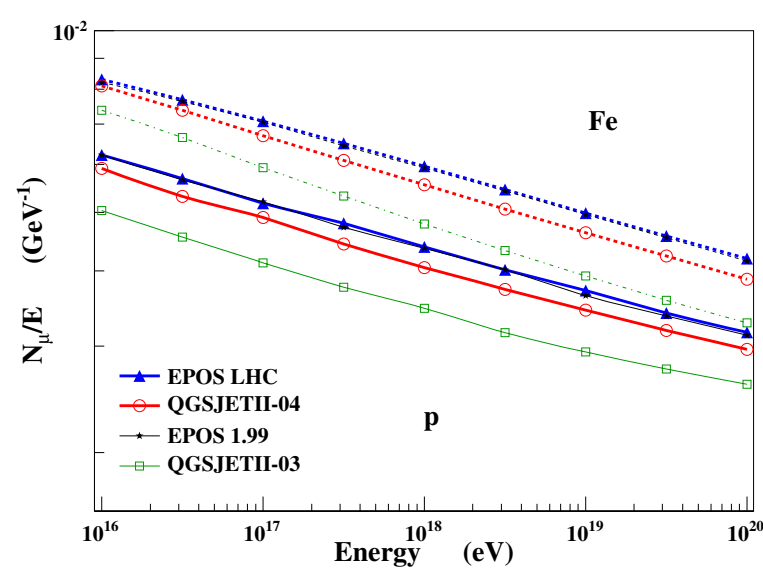

Figure 13. Mean number of muons at ground divided by the primary energy for proton and iron induced showers as a function of the primary energy. Predictions of different highenergy hadronic interaction models: full lines for proton and dashed lines for iron with full stars for EPOS 1.99, open squares for QGSJETII-04, open circles for QGSJETII-03, and full triangles for the results of EPOS LHC.

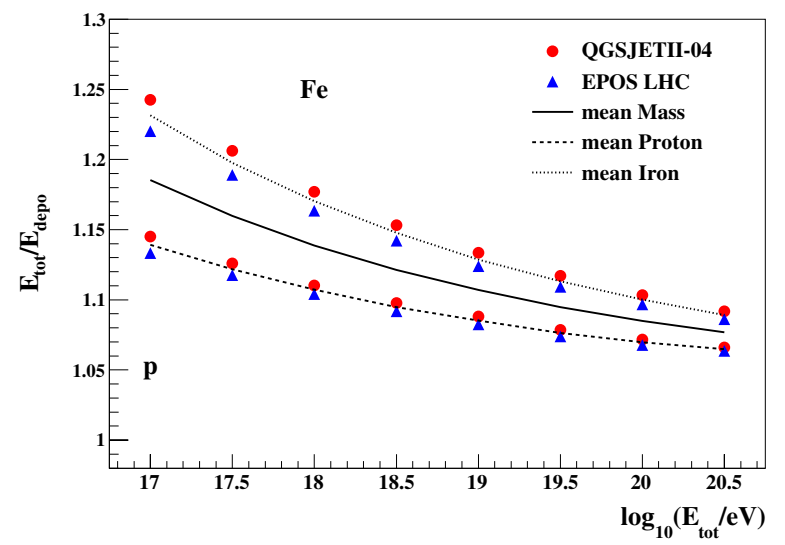

Figure 14. Inverse of missing energy factor for proton and iron induced showers as a function of the primary energy. Predictions of different high-energy hadronic interaction models are shown with triangles for EPOS LHC and circles for QGSJETII-04. Lines are averages between models.

muons in the atmosphere. The average energy of the muons is, for the moment, larger in QGSJETII-04 than in EPOS.

A consequence of the larger mean energy of the muons in EAS simulated with QGSJETII-04 is that the missing energy is larger for showers simulated with this model even if the total number of muons is larger in EPOS LHC. EPOS LHC results are very similar to those of EPOS 1.99. The factor to correct for the missing energy in fluorescence based telescopes is presented in Fig. 14. The difference between QGSJETII-04 and the previous version QGSJETII-03 frequently used as reference in the past is less than $3 \%$ but it has a direct influence on the energy scale considered to compare simulations and data. It should not be neglected.

\section{MPD measurements}

We have seen in the previous section how LHC data could improve the description of EAS using updated hadronic interaction models. But in fact in one particular

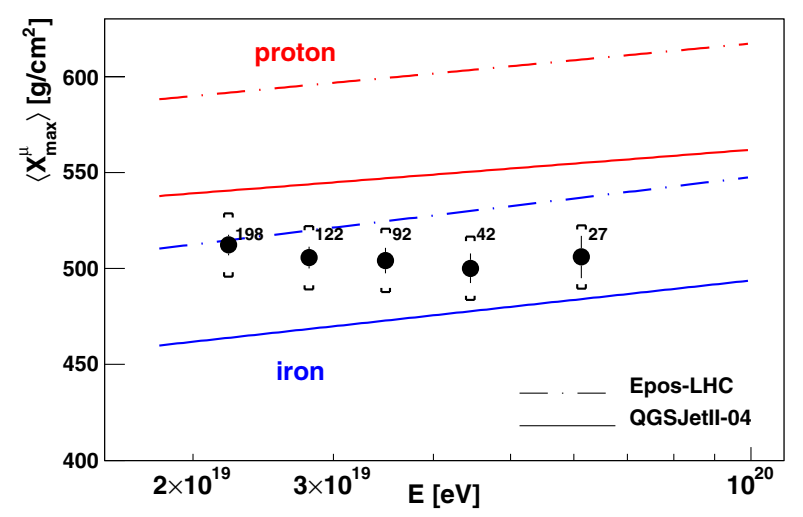

Figure 15. $\left\langle X_{\max }^{\mu}\right\rangle$ as a function of energy from [49]. The predictions of different hadronic models for protons and iron are shown. Numbers indicate the number of events in each energy bin, and brackets represent the systematic uncertainty.

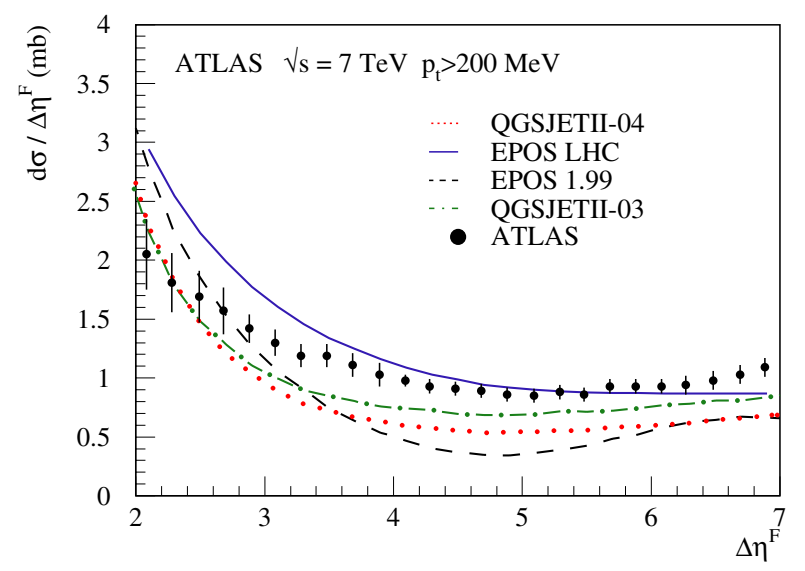

Figure 16. ATLAS measurement of the pseudorapidity gap $\Delta \eta^{F}$ for particles with $p_{t, \text { cut }}>200 \mathrm{MeV}$ in minimum bias events at $7 \mathrm{TeV}$ [51] compared to EPOS LHC (solid line), EPOS 1.99 (dashed line), QGSJETII-04 (dotted line) and QGSJETII-03 (dash-dotted line) simulations.

case, the update of EPOS leads to inconsistent results: the muon production depth measured by the Pierre Auger Observatory [49]. As shown in Fig. 15 the data are out of the range defined by the proton and iron primary mass when EPOS LHC is used for the simulation while QGSJETII-04 gives consistent results. In a previous analysis [50], EPOS 1.99 was giving consistent results, so the important shift observed in MPD simulated with EPOS LHC can easily be identified as a consequence of the change in elasticity due the corrections in diffractive interactions needed to reproduce the rapidity gap distributions measured by the ATLAS collaboration [51]. We can see in Fig. 16 that none of the pre-LHC models could describe the large rapidity gap cross-section, while EPOS LHC gives reasonable results.

The change of the parameters needed to describe the rapidity gap correctly (the diffractive cross-section and the diffractive mass distribution) affected both proton and pion interactions because the same parameters were used for both types of projectile. While the change of diffraction and thus of elasticity in proton interaction has very little impact on $\left\langle X_{\max }^{\mu}\right\rangle$, it appears that MPD are extremely 


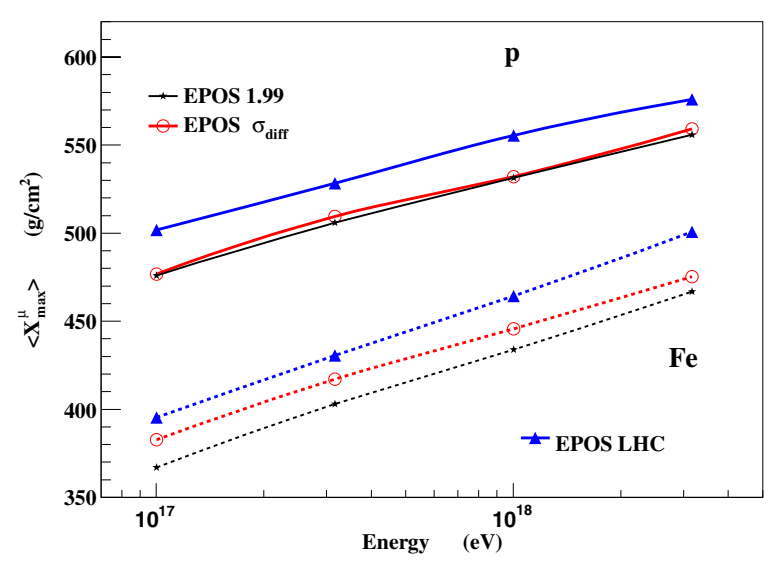

Figure 17. $\left\langle X_{\max }^{\mu}\right\rangle$ as a function of energy for EPOS LHC (triangles), EPOS 1.99 (stars) and EPOS LHC $\sigma_{\text {diff }}$ with a reduced elasticity in pion-air interactions (open circles).

sensitive to the elasticity of pion interactions. This can be understood by the fact that muons are produced at the end of the hadronic cascade after many generations of mainly pion-air interactions. As a consequence of this cumulative effect, even a small increase of only about $10 \%$ of the elasticity of pion-air interactions can lead to large shift in $\left\langle X_{\max }^{\mu}\right\rangle$.

To check this hypothesis, the diffractive cross-section for pion interactions has been reduced in EPOS LHC to get a reduction of about $10 \%$ of the elasticity of the pion-air interactions. As a result $\left\langle X_{\max }^{\mu}\right\rangle$ is reduced by about $30 \mathrm{~g} / \mathrm{cm}^{2}$ and is very similar to the one obtained using EPOS 1.99 as shown in Fig. 17. The diffraction has not been changed for proton interactions to keep full compatibility with LHC data.

Such a small change is compatible with all pionnucleus data that are available at low energy and thus these two versions of EPOS cannot be discriminated from accelerator data. But the effect on MPD is so strong that data from the Pierre Auger Observatory can be used to constrain diffraction in pion interactions to get consistent results between the mean logarithmic mass which can be extracted from $\left\langle X_{\max }^{\mu}\right\rangle$ and the one deduced from $\left\langle X_{\max }\right\rangle$ which has very little dependence on pion hadronic interaction (cf. Sect. 2.2). From EAS development we can thus say that the elasticity of pion-air interactions should be lower than the elasticity of proton-air interactions.

\section{Summary}

Using a simple cascade model, it is possible to find the main parameters of hadronic interactions that influence air shower predictions. For the mean depth of shower maximum, $\left\langle\mathrm{X}_{\max }\right\rangle$, these parameters are the inelastic cross sections, the secondary particle multiplicity, and the inelasticity (not studied here). Using recent LHC data at $7 \mathrm{TeV}$ it is possible to reduce the uncertainty in the extrapolation of the hadronic interaction models used for EAS simulations. Using pre- and post-LHC versions of the QGSJETII and EPOS models, it has been shown that the difference in multiplicity between these models has been reduced by a factor of 5 at the highest energy, resulting in a very similar elongation rate. There is still a systematic shift in $X_{\max }$ of about $20 \mathrm{~g} / \mathrm{cm}^{2}$ due to remaining differences in the multiplicity (and elasticity) of the models. This uncertainty is comparable to the experimental uncertainty in the measurement of $X_{\max }$. As a consequence the interpretation of the data using postLHC data will be more reliable, especially concerning the possible change in mass composition with energy as summarized in [52].

For the number of muons, the ratio between particles producing hadronic sub-showers and the total number of particles is very important. LHC data are important to constrain (anti)baryon and strangeness production at midrapidity. Lower energy data of fixed target experiment are also important to measure forward production of $\pi^{0}$ for instance. Taking into account both aspects, the new version of the QGSJETII and EPOS models predict very similar results close to EPOS 1.99 model, but with harder spectrum.

The difference between EPOS 1.99 and the results of EPOS LHC is not very large because most of the changes are taking place at mid-rapidity. This phase space is good to test the physics of the model but is not very important for air shower development. A contrario, large differences between QGSJETII-03 and QGSJETII-04 are observed. With a larger $\left\langle\mathrm{X}_{\max }\right\rangle$ the average mass is heavier than before at Auger energies. In addition the number of muons increased by about $20 \%$ to a value closer to EPOS 1.99 but with a larger mean energy for the muons at ground level.

Air shower measurements, such as the muon production depth, can also give very strong constraints on hadronic interactions in particular for pion interactions for which cumulative effects due to the hadronic cascade can be observed.

To conclude, we can say that LHC data contribute a lot to reducing the uncertainties in air shower simulations, providing better tools to analyze cosmic ray data. The differences between the hadronic models have been reduced but one should keep in mind that there are still uncertainties in the models themselves which have to be better quantified and transferred to the calculation of the systematic errors in EAS analysis. Also the consistency of different EAS observables can and should be used to test the hadronic interaction models.

The author would like to thank the Pierre Auger Collaboration for useful discussions.

\section{References}

[1] J. Blümer, R. Engel, J.R. Hörandel, Prog. Part. Nucl. Phys. 63, 293 (2009), 0904.0725

[2] J. Knapp, D. Heck, S.J. Sciutto, M.T. Dova, M. Risse, Astropart. Phys. 19, 77 (2003), astro-ph/0206414

[3] T. Antoni et al. (KASCADE), Astropart. Phys. 16, 245 (2002), astro-ph/0102443

[4] T. Antoni et al. (KASCADE), Astropart. Phys. 24, 1 (2005), astro-ph/0505413

[5] M. Amenomori et al. (Tibet AS $\gamma$ ), Phys. Lett. B632, 58 (2006), astro-ph/0511469 
[6] T. Abu-Zayyad et al. (HiRes-MIA), Phys. Rev. Lett. 84, 4276 (2000), astro-ph/9911144

[7] J. Abraham (Pierre Auger Collaboration), Phys. Rev. Lett. 104, 091101 (2010), 1002.0699

[8] R.U. Abbasi et al. (HiRes), Phys. Rev. Lett. 104, 161101 (2010), 0910.4184

[9] J. Knapp, D. Heck, G. Schatz (1996), in Wissenschaftliche Berichte FZKA 5828, Forschungszentrum Karlsruhe

[10] M. Zha, J. Knapp, S. Ostapchenko, Proc. of 28th Int. Cosmic Ray Conf., Tsukuba p. 515 (2003)

[11] R. Ulrich, R. Engel, M. Unger (2010), 1010.4310

[12] R. Engel, D. Heck, T. Pierog, Ann. Rev. Nucl. Part. Sci. 61, 467 (2011)

[13] B. Alessandro, D. Bergman, M. Bongi, A. Bunyatyan, L. Cazon et al. (2011), 1101.1852

[14] D. d'Enterria, R. Engel, T. Pierog, S. Ostapchenko, K. Werner, Astropart. Phys. 35, 98 (2011), 1101.5596

[15] T. Bergmann et al., Astropart. Phys. 26, 420 (2007), astro-ph/0606564

[16] W. Heitler, Quantum Theory of Radiation (Oxford University Press, Oxford, 1944), 2nd edition

[17] J. Matthews, Astropart. Phys. 22, 387 (2005)

[18] C. Meurer, J. Blümer, R. Engel, A. Haungs, M. Roth, Czech. J. Phys. 56, A211 (2006), astro-ph/0512536

[19] J. Alvarez-Muniz, R. Engel, T.K. Gaisser, J.A. Ortiz, T. Stanev, Phys. Rev. D66, 033011 (2002), astro-ph/0205302

[20] T. Pierog, K. Werner, Phys. Rev. Lett. 101, 171101 (2008), astro-ph/0611311

[21] S. Ostapchenko, Phys. Rev. D74, 014026 (2006), hep-ph/0505259

[22] S. Ostapchenko, Phys. Lett. B636, 40 (2006), hep-ph/0602139

[23] S. Ostapchenko, Phys. Rev. D83, 014018 (2011), 1010.1869

[24] K. Werner, F.M. Liu, T. Pierog, Phys. Rev. C74, 044902 (2006), hep-ph/0506232

[25] T. Pierog, K. Werner, Nucl. Phys. Proc. Suppl. 196, 102 (2009), 0905.1198

[26] D. Heck, J. Knapp, J. Capdevielle, G. Schatz, T. Thouw (https://web.ikp.kit.edu/corsika/), Wissenschaftliche Berichte, Forschungszentrum Karlsruhe FZKA 6019 (1998)

[27] T. Csörgö et al. (TOTEM Collaboration), Prog. Theor. Phys. Suppl. 193, 180 (2012), 1204.5689

[28] R. Engel, H. Rebel, Acta Phys. Polon. B35, 321 (2004)
[29] T. Pierog, I. Karpenko, J. Katzy, E. Yatsenko, K. Werner (2013), 1306. 0121

[30] H. J. Drescher, M. Hladik, S. Ostapchenko, T. Pierog, K. Werner, Phys. Rept. 350, 93 (2001), hep-ph/0007198

[31] F. Liu, J. Aichelin, M. Bleicher, H. Drescher, S. Ostapchenko et al., Phys. Rev. D67, 034011 (2003), hep-ph/0202008

[32] M. Bleicher, F. Liu, A. Keranen, J. Aichelin, S. Bass et al., Phys. Rev.Lett. 88, 202501 (2002), hep-ph/0111187

[33] M. Hladik, H.J. Drescher, S. Ostapchenko, T. Pierog, K. Werner, Phys. Rev. Lett. 86, 3506 (2001), hep-ph/0102194

[34] K. Werner, Phys. Rev. Lett. 98, 152301 (2007), 0704.1270

[35] D.S. Barton et al., Phys. Rev. D27, 2580 (1983)

[36] N.N. Kalmykov, S.S. Ostapchenko, A.I. Pavlov, Bull. Russ. Acad. Sci. Phys. 58, 1966 (1994)

[37] S. Ostapchenko, Phys. Rev. D81, 114028 (2010), 1003.0196

[38] H. Jung et al. (2009), 0903. 3861

[39] C. Caso et al. (Particle Data Group), Eur. Phys. J. C3, 1 (1998)

[40] F. Abe et al. (CDF), Phys. Rev. D41, 2330 (1990)

[41] V. Khachatryan et al. (CMS), Phys. Rev. Lett. 105, 022002 (2010), 1005. 3299

[42] K. Aamodt et al. (ALICE Collaboration), Eur. Phys. J. C68, 345 (2010), 1004. 3514

[43] M. Chojnacki (ALICE Collaboration), J. Phys. G38, 124074 (2011), 1109.6744

[44] S. Chatrchyan et al. (CMS Collaboration), Eur. Phys.J. C72, 2164 (2012), 1207.4724

[45] M. Ataian et al. (EHS-NA22 Collaboration), Z. Phys. C54, 247 (1992)

[46] N. Agababyan et al. (EHS-NA22 Collaboration), Z. Phys. C46, 387 (1990)

[47] N. Abgrall et al. (NA61/SHINE Collaboration), Phys. Rev. C84, 034604 (2011), 1102.0983

[48] O. Adriani et al. (LHCf Collaboration), JINST 3, S08006 (2008)

[49] A. Aab et al. (Pierre Auger Collaboration), Phys. Rev. D90, 012012 (2014), 1407.5919

[50] P. Abreu et al. (Pierre Auger Collaboration), Proceeding of the 32nd ICRC, Beijing, China (2011), 1107.4804

[51] G. Aad et al. (ATLAS Collaboration), Eur. Phys. J. C72, 1926 (2012), 1201.2808

[52] K.H. Kampert, M. Unger, Astropart. Phys. 35, 660 (2012), 1201.0018 